\title{
Towards a Sophisticated Understanding of Service Design for Enterprise Architecture
}

\author{
Stephan Aier and Bettina Gleichauf \\ Institute of Information Management, University of St. Gallen, \\ Müller-Friedberg-Strasse 8 \\ 9000 St. Gallen, Switzerland \\ \{stephan.aier, bettina.gleichauf\}@unisg.ch
}

\begin{abstract}
The service orientation approach emerged form the software engineering community and has now become a widely discussed design paradigm for almost every aspect of an enterprise architecture (EA). However, experience from cases studies has shown that it is necessary to explicitly differentiate service categories in EA, its goals and its resulting design guidelines. This paper derives a sophisticated understanding of different services categories, their respective goals and design guidelines based on empirical research.
\end{abstract}

\section{Introduction}

Enterprise architecture (EA) describes the fundamental structure of an organization $[22,25,29,33]$ and supports an organization's transformation by offering a holistic perspective on their elements and the relations between these elements [18]. EA is widely accepted as an approach to manage transformations by (a) propagating strategy and organizational changes consistently to software and infrastructure descriptions, by (b) supporting consistent business transformation enabled by technology innovations, and by (c) decoupling business-oriented and technology-oriented partial architectures $[1,9,13,23,30,32]$. The main challenges of this transformation are the complexity of existing structures, the interdependencies of the architectural elements as well as the heterogeneity of ownership of architectural elements within an organization. The goals that are addressed by EA programs aiming at these challenges are transparency, consistency, IT/Business alignment, stakeholder orientation, standardization, reuse and eventually flexibility and sustainability.

Service orientation as a paradigm not only for software engineering but for enterprise architecture engineering addresses these goals and challenges [26, 27]. However, service orientation precisely discussed in software engineering, e.g. [6, 10, 34], has developed to a broad discussion concerning almost all architectural layers from strategy and business architecture to software, data and infrastructure architecture [33]. Unfortunately this discussion often lacks the necessary precision e.g. when discussing non-technical aspects of service orientation.

When service orientation is discussed as a design paradigm for EA it is indispensable to explicitly differentiate the design goals and their resulting design principles for different design objects in an EA since experience from case studies shows that there is hardly any "one-size-fits-all" design methodology that is equally effective for 
e.g. the design of technology related services as well as business architecture related services at the same time.

Therefore the research questions of this paper are: (a) Which kinds of services should be differentiated from an EA perspective? (b) Which goals are related to these service categories and what are the consequences for service design? To answer these questions we outline three industry case studies to find similarities in their understanding and definitions of services. Based on these cases studies we derive our understanding of services categories in an EA as well as the related goals and design principles for each category.

\section{Related Work}

Within the last years services were mainly discussed with regard to the technical design of (software) services. There is a multitude of literature concerning this topic and dealing with the actual realization of service orientation, e.g. in the form of web services (e.g. [6, 10, 34]). Besides the realization through software services several authors also claim a comprehensive understanding of service orientation on business layers to the point of a service oriented enterprise [7, 8, 27]. The term "enterprise services" is often used in this context. However, until today there is no consensus either on a clear definition and conceptual delimitation of such services on business layers opposed to software services or among one another or on possible subtypes of these services.

Literature sources say little on the differentiation between the goals that are pursued by the different types of services. Among the general goals mentioned in connection with software services are improvement of process support and increased process flexibility [6], agility of the information systems [11], cost savings due to low maintenance IT systems as well as higher quality due to the possible reuse of software components [7, 16]. In terms of the design of services reuse is acknowledged as the main purpose [14]. LEGNER and HEUTSCHI further identify the goals standardized integration infrastructure and decoupling of application domains [19]. This shows the tight linkage of service orientation and EA. Service orientation can serve as a common "language" between IT and business as it is a fundamental paradigm [18]. The main impact of SOA on EA is the enhanced flexibility in terms of new business partners, new processes, new assets, all while not affecting business operations or enforcing the implementation of a new EA model. Despite these obvious benefits of software service design, there is still no evidence on how these goals can be applied to develop guidelines for the design of services on the business layers of an EA.

With regard to service design most authors refer to the significance of interface orientation and, in conjunction, of service interoperability [11]. Additionally, LEGNER and HEUTSCHI identify the design principles of autonomy and modularity as well as business suitability from existing SOA research on the design of software services [19]. Several authors have presented ideas how enterprise services in particular can be identified and designed $[15,24,27]$ but they still remain very close to the concept of software services that are used in a business context. Therefore they lack a business perspective on service design for a comprehensive EA. SCHELP and WINTER show that the conceptual design of enterprise services does not differ considerably from 
traditional application design. However they point out that a well-founded and differentiated design method for enterprise services is still missing [28].

Considering the diverse focus of the different service types within a comprehensive EA, the need for differentiated guidelines for services construction becomes evident: In order to adequately apply the required business perspective to business architecture related services and effectively pursue the resulting goals, the construction of such services will differ substantially from software service design. Corresponding design guidelines should be derived from the goals pursued, which have to be identified and structured first. Therefore the paper at hand examines the diversity of service types from an EA perspective and shows how they can be designed in a differentiated way.

\section{Case Studies}

For deriving a set of differentiated types of services, to describe their design goals as well as their design rules this section presents three case studies. The case studies are used to consolidate essential characteristics of the differentiated service definitions observed. Data for the case studies have been collected with two of these companies since 2002/2003 and with the remaining one since 2006. Key stakeholders in IT management, architecture management (i.e. IS and business architects), and Business/IT relationship management have been interviewed. In addition to the interviews regular review meetings have been set up to observe state, development, and architectural issues in the companies involved. The companies participated in long term collaborative research projects in IS integration and enterprise architecture involving ten companies in the period of 2002-2008. The companies chosen for this study have a long term experience with service oriented architectures and have mature architecture management structures in place. Data presented in the case studies below aggregate the research results gained with these companies until summer 2008. Due to company request the case studies have been made anonymous.

\subsection{Company A}

Company A is an IT service provider for a large banking network in Germany. In its current form the network is the result of several mergers of formerly independent and regionally organized IT service providers. Every formerly independent company had its own evolutionary grown banking solution. However, none of these solutions had a predominant position within the network. Therefore the network decided to implement a new and common system as their core banking solution. The development started in 2002 and was finished in 2005 for the time being. The new system is designed following a service oriented paradigm in order to adapt and to consistently provide the implemented functionality to every partner.

On a business level the enterprise architecture of company A is designed following the network's process reference model. The process reference model serves as a structural blueprint for the design of the actual business processes consisting of several steps, e.g. choosing a certain product for a credit application. Single steps of a process are designed as enterprise services. These enterprise services are eventually implemented as software services on a system level in the enterprise architecture. 
Each enterprise service, e.g. management of a credit application, may be used in the entire network for a broad range of products. Throughout the network, reuse of enterprise services is explicitly intended. An enterprise service is comprised of a selfcontained set of business functionalities and belongs to a specific domain but may also be reused in other domains. Each enterprise service is linked with exactly one software service as a technical implementation. However, software services may be called in different contexts which may result in a different behaviour.

\subsection{Company B}

Company B is one of the largest insurance companies in Switzerland. They have started their first projects utilizing a service oriented software design at the end of the 1990ies with the introduction of web applications which integrated basic functionalities of the host systems. These early projects aimed at providing functionality over internet technology. Effects like reuse occurred rather accidentally. However, the potential of service oriented design has soon been recognized and resulted in standardization initiatives as well as an embedment in the company's enterprise architecture framework in order to systematically foster reuse and maintainability of services.

Company B differentiates three layers of service orientation in their architecture: A user-access layer, a process layer and a service layer. The service layer contains business activity services which call business object services. Business object services directly access software systems and may run an update on a database record for example. The process layer does not explicitly define process services but it contains business processes, sub-processes as well as detailed workflow definitions. Workflows employ the functional specifications of the business activity services but may also access business object services directly. On the top level access to application's graphical user interfaces is designed by employing access services implemented in e.g. portals.

The variety of possibilities to use the service framework of company B provides a high flexibility and enables the adaptation to a variety of situations. However, it also demands for strong governance in order to preserve the maintainability of such a framework.

\subsection{Company $\mathrm{C}$}

Company $\mathrm{C}$ is a globally operating telecommunications service provider with a large, complex and heterogeneous application landscape. At the end of the last century, corporate management decided to structure the corporate group into four rather independent divisions representing the four strategic business areas. The new structure reduced the overall complexity by reducing the interdependencies between the four divisions on a business layer as well as on a technology layer by definition. At the same time, however, the heterogeneity as well a redundancy between the divisions grew as a result of their new independence. This independence resulted in e.g. inconsistent information about customers where different divisions served the same customer segments with different, division-specific products. As a consequence, divisions have been continually integrated again in order to leverage synergies among them.

Company $\mathrm{C}$ primarily focuses the definition of enterprise services as a solid and standardized definition of business functionalities. The major goal of this standardization is to 
provide a reusable repository of enterprise services in order to enable the flexible and fast definition of new or changed products. Consequently the initial identification of enterprise services will be derived from product definitions, e.g. an availability check for an internet connection. For the actual execution the enterprise services employ software services which implement the necessary functionality, e.g. the measurement of signal quality on the physical wire. The encapsulation of required functionality in enterprise services provides the necessary decoupling of the product layer and the technical software layer.

\section{Differentiating Services and Guidelines for Service Construction}

Contemplating these case studies two categories of services can clearly be identified: On a system layer software services carry out the technical realization of functional tasks while enterprise services (or business activity services in company B) encapsulate functionalities. At this point it is crucial to determine that the term enterprise services does not stand for pieces of software-as opposed to the wording e.g. in [24] — but it denotes model based abstractions. Furthermore the arrangement of services in the case studies leads to the assumption that there is another relevant layer above the enterprise services. Processes and products are located on this layer and are being served by enterprise services (companies A and C).

Architecture

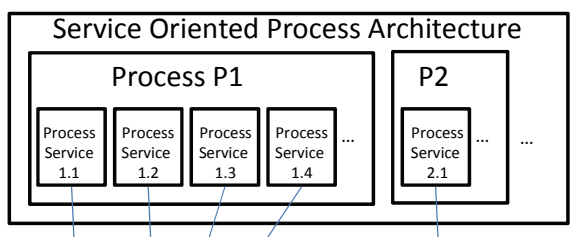

Service Oriented/ntegration Architecture
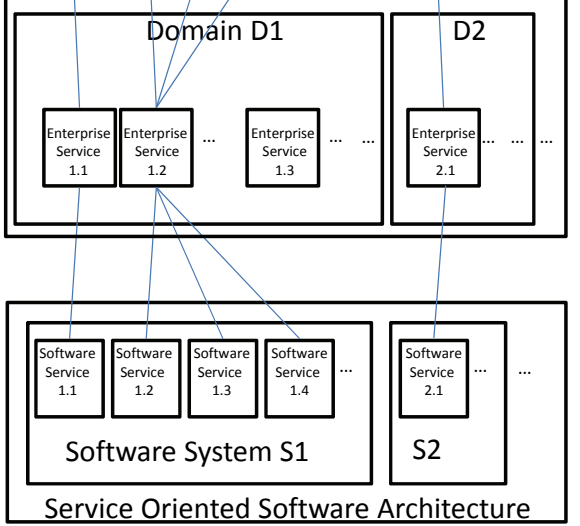

Goals

Design Guidelines

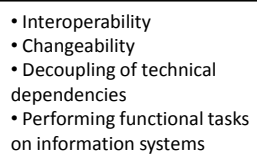

- Interoperability

- Changeability

- Decoupling of technical

dependencies

- Performing functional tasks

on information systems
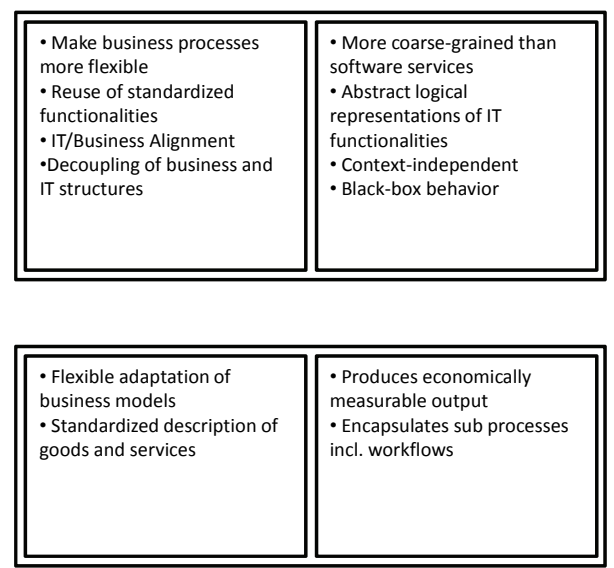

- Along data objects affected - As granular as necessary

Fig. 1. System of differentiated services following [2] 
Taking into account these three layers described by the case studies and an enterprise architecture described by [33], three sub architectures can be distinguished: a Service Oriented Software Architecture (SOSA) on the basis of software services, a Service Oriented Integration Architecture (SOIA) on the basis of enterprise services and a Service Oriented Process Architecture (SOPA) on the basis of process services (Figure 2).

\subsection{Service Oriented Software Architecture}

The goals of a SOSA are interoperability and changeability on the software layer by the means of software services. Changeability and flexibility should be achieved by decoupling, i.e. by the reduction of technical dependencies. Moreover, software services in a SOSA are used to perform functional tasks on information systems [3].

In order to realize decoupling, the design of software services should be orientated to the data object produced, changed or consumed by them. This is also done in practice like all of the three case studies show: Software services are designed in order to conduct functional tasks on the supporting information systems. There already exists a lot of literature on how to design such software services (cf. chapter 2). According to the goals of a SOSA loose coupling and interoperability are the main guidelines for software services design. Concerning the granularity of software services, our research findings show that services should be as granular as necessary in order to support the functional tasks.

Depending on the task to be performed component services (context oriented services), process services, entity services (data oriented services) and utility services can be identified [24]. For their SOA blueprints OASIS distinguishes between atomic, independent services and composite services. Regarding their statefulness and specific task conversational services, data services, publish-subscribe services and service brokers can be named [21].

The adoption of software services is reasonable in highly volatile environments because interoperability and loose coupling provide advantages in implementation time [16]. On the other side, one finding from our research was that the effectiveness of service orientation in areas that claim extremely high standards concerning performance, transactionality or data security have to be analyzed in detail first (cf. also security and transaction issues discussed in [20]).

\subsection{Service Oriented Integration Architecture}

A SOIA aims at the creation of enterprise services in order to orchestrate business processes more flexibly. Besides flexibility reuse of standardized functionalities is named as a key objective in the case studies presented above. Company $\mathrm{C}$ indirectly aims at process flexibility by concentrating on products as a result of business processes. SCHELP and WINTER [28] mention the connection of business-oriented artifacts and IT artifacts as the main objective of service orientation on the integration layer. Thus, enterprise services aim at supporting IT/Business Alignment. With regard to the alignment objective of enterprise services, case study $\mathrm{C}$ seems especially interesting: Enterprise services are applied in order to establish a stable "middle layer" that is able 
to realize the relations between the fast changing products on one side and the longterm IT systems on the other side. Besides flexibilisation, reuse and IT/Business Alignment SOIA focuses on the decoupling of different change frequencies of business and IT structures.

In order to operationalize these objectives, enterprise services should provide a discontinuation of interdependencies between functional and technical structures. Therefore, enterprise services should be more coarse-grained compared to the software services underneath and the process services above [2]. Company $\mathrm{C}$ is realizing this principle. In company A each enterprise service is assigned to a software service, though the access is possible through different call options. Thus the coarsening between software services and enterprise services is implemented indirectly.

Enterprise services can be defined as abstract logical representations of IT functionalities on the integration layer [28]. To enable flexibilisation of functionalities within business processes, enterprise services should encapsulate coarse-grained functionalities [2]. In contrast to activities enterprise services should be able to exist without contextual information on business processes [31], thus they should not contain information on workflows or the like. Another crucial service design principle in view of the IT/Business Alignment and the flexibilisation of processes is a black box behavior of the services.

KOHLMANN and Alt [15] as well as SCHELP and WINTER [27] present approaches for the design of enterprise services: KOHLMANN and ALT [15] propose a businessdriven identification of enterprise services by deducing them from reference processes while considering role models. The orientation towards processes, functions and data objects as well as the aggregation of self-contained activity sets is also named by SCHELP and WINTER [27] among the main design guidelines for enterprise services. This approach seems to be applicable in practice, too: Company A identifies enterprise services by the means of given reference processes while company B is deducing the specification for enterprise services directly from their business activities and business objects that have to be realized.

Enterprise services can be differentiated in loose coupled, i.e. orchestrated, or tightly coupled, i.e. composed services [27]. KOHLMANN and ALT [15] distinguish between enterprise service types for process activities, business rules and business objects. Company B also models activity oriented and business object oriented services. According to the traditional application design SCHELP and WINTER [28] distinguish product centered, information centered and function centered enterprise services. In practice the differentiation along contextual factors seems sensible: In company A enterprise services are assigned to application domains so the services are grouped by explicit business contexts. This may lead to the differentiation in internal or external services.

In order to successfully apply service orientation to functionalities the decomposition of functionalities should be logically possible. The presented companies A, B and $\mathrm{C}$ only partition and encapsulate functionalities if they affect multiple business objects, like customers or business processes, so that reuse is possible. If the decomposition in services results in high complexity and reuse potentials are no longer given, service orientation should not be applied in this business area. For example Company A does not design singular functionalities by the service orientation paradigm. 


\subsection{Service Oriented Process Architecture}

On the organization or process layer, service orientation can be used to flexibly adapt business models by the means of process services. An example for the need of such an adaptation is the outsourcing of individual business functions or processes (business process outsourcing). The essential explication of service interfaces makes the shift of goods and services into another environment possible. By defining process services a structured description of goods and services is achieved at the same time.

Process service should encapsulate sub processes respectively a self-contained activity set that has an economically measurable output. Opposed to enterprise services process services also incorporate sub processes' workflows that are deduced from existing process models that exist in the company. In order to anticipate the possible changes in the process design, like outsourcing or inter-corporate collaboration, the design of process services should consider the usage of non-exclusive, common standards [4].

The delimitation of process services as opposed to enterprise services on the integration layer seems to be tough in practice, as our case studies show: In company A individual process steps are identified but not explicitly implemented as a service. Company B differentiates between business activities and business objects but as regards content they are rather instances of enterprise services than process services.

In view of the flexibility for new business models the implementation of process services seems reasonable if the sub processes can be standardized concerning their quality and execution.

\section{Discussion}

This contribution illustrates the necessity to precisely and explicitly differentiate service categories from an EA perspective. The main reasons to differentiate services are the different goals and resulting different design guidelines applying to these service categories.

Based on three cases studies we differentiate software services, enterprise services and process services. While the cases studies and also literature provide a profound understanding of software services and enterprise services, the design of process services is dominated by a classical top-down process design. The fact that classical business process management and process modelling predominantly use a top-down approach while service orientation traditionally aims at clustering existing assets applying a bottom-up methodology may lead to new challenges but also to new synergies. In process modelling research, reuse of process building blocks has also been a topic discussed for several years, often in conjunction with (process) reference models (e.g. $[5,17])$. It can be supposed that such approaches are relevant and fruitful for process service design. Therefore, future research should elaborate on the appropriateness of "classical" process design in a services oriented EA. In doing so, the applicability and the practical use of SOPA can be further evaluated.

An opportunity for further research can also be found in the field of service design guidelines. Neither literature nor the practitioner's community provide an empirically validated understanding of which design guidelines effectively contribute to their 
respective goals. There are only a few publications on service design guidelines-and only as far as IT architecture is concerned. Regarding integration and process architecture, there is hardly any publication on service design. Empirical validation of successful service design guidelines-especially for service oriented integration and process architecture - is non-existent and practitioners' experiences are limited. However, service design guidelines which have proven to be successful will be beneficial for the practitioners' community and therefore represent a reasonable goal for explorative research.

We strongly encourage research which investigates the influence of contextual factors on the success of services orientation. The contingency theory of leadership [12] argues that there is no "best way" of organizing or leading an organization. Instead, there are various internal and external factors that influence organizational effectiveness. The organizational style must therefore be contingent upon those factors. Translated into the context of success of service orientation, it stands to reason that contingency factors such as industry sector, organizational size or prior experience with service orientation might influence SOA success.

Finally, for a validation of the concept of different service sub-architectures, the interplay of SOPA, SOIA and SOSA should be analyzed in detail. It can be assumed that the relationships between the different services on different layers are quite complex - both, as EA models and as their observable artifacts. In order to describe and to establish a comprehensive and flexible EA based on services, the links between the different layers need to be understood thoroughly.

\section{References}

1. Aier, S., Riege, C., Winter, R.: Classification of Enterprise Architecture Scenarios - An Exploratory Analysis. Enterprise Modelling and Information Systems Architectures 3(1), 14-23 (2008)

2. Aier, S., Winter, R.: Virtual Decoupling for IT/Business Alignment - Conceptual Foundations, Architecture Design and Implementation Example. Business \& Information Systems Engineering 51(2) (forthcoming, 2009)

3. Alonso, G., Casati, F., Kuno, H., Machiraju, V.: Web Services - Concepts, Architctures and Applications. Springer, Heidelberg (2004)

4. Alt, R., Fleisch, E.: Key Success Factors in Designing and Implementing Business Networking Systems. In: Proceedings of Proceedings of 12th Electronic Commerce Conference: Global Networked Organizations, Bled, Slovenia, Kranj, Slovenia, Moderna organizacija, June 7-9, 1999, pp. 219-235 (1999)

5. Baacke, L., Rohner, P., Winter, R.: Aggregation of Reference Process Building Blocks to Improve Modeling in Public Administrations. In: Proceedings of Electronic Government, 6th International EGOV Conference, Proceedings of ongoing research, project contributions and workshops, Trauner Druck, pp. 149-156 (2007)

6. Berbner, R., Grollius, T., Repp, N., Eckert, J., Heckmann, O., Ortner, E., Steinmetz, R.: Management of Service-oriented Architecture (SOA)-based Application Systems. Enterprise Modelling And Information System Architectures 2(1), 14-25 (2007)

7. Bieberstein, N., Bose, S., Walker, L., Lynch, A.: Impact of Service-oriented Architecture on Enterprise Systems, Organizational Structures, and Individuals. IBM Systems Journal 44(4), 691-708 (2005) 
8. Brown, G., Carpenter, R.: Successful Application of Service-Oriented Architecture Across the Enterprise and Beyond. Intel Technology Journal 8(4), 345-359 (2004)

9. Buchanan, R.D., Soley, R.M.: Aligning Enterprise Architecture and IT Investments with Corporate Goals, Object Management Group (2002)

10. De Backer, M., Dedene, G., Vandelbulcke, J.: Web Services Composition, Execution and Visualization. In: Proceedings of Proceedings of the 12th IEEE International Workshop on Program Comprehension (IWPC 2004), pp. 264-265. IEEE Computer Society Press, Los Alamitos (2004)

11. Erl, T.: SOA: Principles of Service Design. Prentice Hall, Upper Saddle River (2007)

12. Fiedler, F.E.: A Contingency Model of Leadership Effectiveness. Advances in Experimental Social Psychology 1, 149-190 (1964)

13. Fischer, R., Aier, S., Winter, R.: A Federated Approach to Enterprise Architecture Model Maintenance. Enterprise Modelling and Information Systems Architectures 2(2), 14-22 (2007)

14. Herr, M., Bath, U., Koschel, A.: Implementation of a Service Oriented Architecture at Deutsche Post MAIL. In: Zhang, L.-J., Jeckle, M. (eds.) ECOWS 2004. LNCS, vol. 3250, pp. 227-238. Springer, Heidelberg (2004)

15. Kohlmann, F., Alt, R.: Business-Driven Service Modeling - A Methodology Approach from the Finance Industry. In: Proceedings of BPSC 2007: Proceedings of the 1st International Working Conference on Business Process and Services Computing, Leipzip, pp. 180-193 (2007)

16. Krafzig, D., Banke, K., Slama, D.: Enterprise SOA - Service-Oriented Architecture Best Practices, The Coad Series. Pearson, Upper Saddle River (2005)

17. Lang, K., Bodendorf, F., Glunde, J.: Framework for Reusable Reference Process Building Blocks. SIGGROUP Bulletin 18(1), 68-70 (1997)

18. Lankhorst, M.: Enterprise Architecture at Work: Modelling, Communication and Analysis. Springer, Berlin (2005)

19. Legner, C., Heutschi, R.: SOA Adoption in Practice - Findings from Early SOA Implementations. In: Proceedings of Proceedings of the 15th European Conference on Information Systems, Relevant rigour - Rigorous relevance (2007)

20. McGovern, J., Sims, O., Jain, A., Little, M.: Enterprise Service Oriented Architectures: Concepts, Challenges, Recommendations. Springer, Dordrecht (2006)

21. OASIS, SOA Blueprints, OASIS (2005) (last access: 08.08.2008), http: / /www.oasis-open.org/committees/download.php/15965/ 05-12-00000.001.doc

22. Rood, M.A.: Enterprise Architecture: Definition, Content, and Utility. In: Proceedings of Proceedings of the Third Workshop on Enabling Technologies: Infrastructure for Collaborative Enterprises, pp. 106-111. IEEE Computer Society Press, Los Alamitos (1994)

23. Ross, J.W., Weill, P., Robertson, D.C.: Enterprise Architecture as Strategy. Creating a Foundation for Business Execution. Harvard Business School Press, Boston (2006)

24. SAP, Enterprise Services Design Guide, SAP AG (2006)

25. Schekkerman, J.: How to Survive in the Jungle of Enterprise Architecture Frameworks: Creating or Choosing an Enterprise Architecture Framework, 2nd edn. Trafford Publishing, Victoria (2004)

26. Schelp, J., Aier, S.: SOA and EA - Sustainable Contributions for Increasing Corporate Agility. In: Proceedings of 42th Hawaii International Conference on Systems Science (HICCS-40). IEEE Computer Society Press, Los Alamitos (2009) 
27. Schelp, J., Winter, R.: Towards a Methodology for Service Construction. In: Proceedings of 40th Hawaii International Conference on Systems Science (HICCS-40). IEEE Computer Society, Los Alamitos (2007)

28. Schelp, J., Winter, R.: Business Application Design and Enterprise Service Design: A Comparison. Int. J. Service Sciences (2008)

29. The Open Group, The Open Group Architecture Framework TOGAF - 2007 Edition (Incorporating 8.1.1), Van Haren, Zaltbommel (2007)

30. Veasey, P.W.: Use of Enterprise Architectures in Managing Strategic Change. Business Process Management Journal 7(5), 420-436 (2001)

31. Vernadat, F.B.: Reengineering the Organization with a Service Orientation. In: Hsu, C. (ed.) Service Enterprise Integration. Integrated Series In Information Systems, vol. 16, pp. 77-101. Springer, Heidelberg (2007)

32. Wagter, R., van den Berg, M., Luijpers, J., van Steenbergen, M.: Dynamic Enterprise Architecture: How to Make It Work. John Wiley \& Sons, Hoboken (2005)

33. Winter, R., Fischer, R.: Essential Layers, Artifacts, and Dependencies of Enterprise Architecture. Journal of Enterprise Architecture 3(2), 7-18 (2007)

34. Yuan, R., Zunchao, L., Boqin, F., Jincang, H.: Architecture-based Web service composition framework and strategy. In: Proceedings of 12th IEEE International Conference and Workshops on the Engineering of Computer-Based Systems (ECBS 2005), pp. 129-134. IEEE Computer Society, Los Alamitos (2005) 